\title{
Traveling wave solutions of new coupled Konno-Oono equation
}

\author{
Md. Abul Bashar, Gobinda Mondal, Kamruzzaman Khan, and Ahmet Bekir ${ }^{1}$ \\ Department of Mathematics, Pabna University of Science and Technology, Pabna, Bangladesh \\ ${ }^{1}$ Department of Mathematics and Computer Science, Eskisehir Osmangazi University, Turkey
}

Received: 13 August 2015, Revised: 14 August 2015, Accepted: 3 April 2016

Published online: 26 May 2016.

\begin{abstract}
In this paper, the recently developed tanh-function method and extended tanh-function method are applied to explore the traveling wave solutions of new coupled Konno-Oono equation. We have successfully derived some explicit traveling wave solutions of new coupled Konno-Oono equation.
\end{abstract}

Keywords: tanh-function method, extended tanh-function method, new coupled Konno-Oono equation, traveling wave solutions

\section{Introduction}

Nowadays NLEEs have become most examined subject of all-embracing studies in several branches of nonlinear sciences. A special class of analytical solutions named traveling wave solutions for NLEEs has a lot of importance, because most of the phenomena that arise in mathematical physics and engineering fields can be described by NLEEs. NLEEs are repeatedly used to describe many problems of protein chemistry, chemically reactive materials, in ecology most population models, in physics the heat flow and the wave propagation phenomena, quantum mechanics, fluid mechanics, plasma physics, propagation of shallow water waves, optical fibers, biology, solid state physics, chemical kinematics, geochemistry, meteorology, electricity, and so forth. Therefore, investigation, traveling wave solutions is becoming more and more attractive in nonlinear sciences day by day. However, not all equations posed of these models are solvable. As a result, many new techniques have been successfully developed by diverse groups of mathematicians and physicists, such as: the Exp-function method [1-5], the Hirota's bilinear transformation method [6,7], the Adomian decomposition method [8], The F-expansion method [9], the Auxiliary equation method [10], $\left(G^{\prime} / G\right)$-expansion method [11-15], the modified simple equation method [16, 17], the tanh-function method [18], the sine-cosine method [19], the improved $\tan (\Phi(\xi) / 2)$-expansion method [20] and many more.

The objective of this paper is to apply the tanh-function method and extended tanh-function method to construct the exact traveling wave solutions for nonlinear evolution: coupled Konno-Oono equations.

\section{Methods}

In this section we will discuss the main steps of tanh-function and extended tanh-function methods. 


\subsection{The tanh-function method}

The tanh-function method summarized by Wazwaz are as follows [21, 22], Suppose a partial differential equation,

$$
P\left(u, u_{t}, u_{x}, u_{x x}, \ldots\right)=0 \text {. }
$$

By using the wave variable $\xi=x-\omega t$ where $U=U(\xi)=U(x, t)$, the equation (1) can be transform to the following ODE:

$$
Q\left(U, U^{\prime}, U^{\prime \prime}, U^{\prime \prime \prime}, \ldots\right)=0
$$

The equation (2) is then integrated as far as all terms contain derivatives. We introducing a new independent variable;

$$
Y=\tanh (\xi)
$$

Equation (3) leading the change of derivatives:

$$
\begin{aligned}
& \frac{d}{d \xi}=\left(1-Y^{2}\right) \frac{d}{d Y} \\
& \frac{d^{2}}{d \xi^{2}}=\left(1-Y^{2}\right)\left(-2 Y \frac{d}{d Y}+\left(1-Y^{2}\right) \frac{d^{2}}{d Y^{2}}\right) \\
& \frac{d^{3}}{d \xi^{3}}=\left(1-Y^{2}\right)\left(\left(6 Y^{2}-2\right) \frac{d}{d Y}-6 Y\left(1-Y^{2}\right) \frac{d^{2}}{d Y^{2}}\left(1-Y^{2}\right)^{2} \frac{d^{3}}{d Y^{3}}\right)
\end{aligned}
$$

The tanh-function method acknowledge the use of the finite expansion

$$
U(\xi)=F(Y)=\sum_{k=0}^{m} a_{k} Y^{k},
$$

where $m$ is a positive integer, for this method, that will be determined. Expansion (7) reduces to the standard tanh-function method [23] for; $1 \leq k \leq m$. The parameter mis comonly attained, as stated before, by balancing the linear terms of the highest order in the resulting equation with the highest order nonlinear terms. If $m$ is not an integer, then a transformation formula should be used to overcome this difficulty. Exchanging (7) into the ODE results an algebraic system of equations in powers of $Y$ that will lead to the determination of the parameters $a_{k}(k=0, \ldots, m)$, and $\omega$. For showing the competency of the method described in the preceding part, we appeard some examples.

\subsection{The extended tanh-function method}

The extended tanh-function method admits the use of the finite expansion [21,22],

$$
U(\xi)=F(Y)=\sum_{k=0}^{m} a_{k} Y^{k}+\sum_{k=1}^{m} b_{k} Y^{(-k)}
$$

where $m$ is a positive integer, for this method, that will be determined. Expansion (8) reduces to the standard tanh-function method for $b_{k}=0 ; 1 \leq k \leq m$. The parameter $m$ is comonly attained, as stated before, by balancing the linear terms of the highest order in the resulting equation with the highest order nonlinear terms. If $m$ is not an integer, then a transformation formula should be used to overcome this difficulty. Exchanging (8) into the ODE results an algebraic system of equations in powers of $Y$ that will lead to the determination of the parameters $a_{k}(k=0, \ldots, m), b_{k}(k=1, \ldots, m)$ and $\omega$. For showing the competency of the method described in the preceding part, we appeared some examples. 


\section{Applications}

In this section we will exert the tanh-function and extended tanh-function methods to find the exact traveling wave solutions then the solitary wave solutions of the new coupled Konno-Oono equations [24] of the form,

$$
u_{x t}-2 u v=0, v_{t}+2 u u_{x}=0 .
$$

We consider the traveling wave transformation equation

$$
u(\xi)=u(x, t), v(\xi)=v(x, t), \xi=x-\omega t
$$

The traveling transformation Eq. (10) transforms Eq. (9) into the following ODEs,

$$
\begin{aligned}
& -\omega u^{\prime \prime}-2 u v=0 \\
& -\omega v^{\prime}+2 u u^{\prime}=0 .
\end{aligned}
$$

Now integrating Eq. (12) with respect to $\xi$, we obtain

$$
v=\frac{1}{\omega}\left(u^{2}+d\right),
$$

where $d$ is an integral constant. Substituting Eq. (13) into the Eq. (11), we attain

$$
\omega^{2} u^{\prime \prime}+2 u d+2 u^{3}=0 .
$$

Now the balancing the highest order derivative $u^{\prime \prime}$ and the nonlinear term $u^{3}$ in Eq. (14), we obtain $m=1$.

\subsection{The tanh-function method}

In this subsection we will apply the tanh-function method to find the exact traveling wave solutions of Konno-Oono equation.

For $m=1$, the finite expansion (7) of tanh-function method admits

$$
u(x, t)=a_{0}+a_{1} Y .
$$

Now, Substituting Eq. (15) into Eq. (14), and equating the coefficients of the powers of $Y$ then we obtain a system of algebraic equations:

$$
\begin{aligned}
& 2 \omega^{2} a_{1}+2 a_{1}^{3}=0, \\
& 6 a_{0} a_{1}^{2}=0, \\
& 6 a_{0}^{2} a_{1}+2 d a_{1}-2 ?^{2} a_{1}=0 . \\
& 2 a_{0}^{3}+2 d a_{0}=0 .
\end{aligned}
$$

By solving these algebraic equations for $a_{0}, a_{1}$ and $\omega$ then we obtain following set of solution:

$$
\omega= \pm \sqrt{d}, a_{0}=0, a_{1}= \pm I \sqrt{d} .
$$


Solving this set of solution, we required the following solutions:

$$
\begin{aligned}
& u(x, t)= \pm I \sqrt{d} \tanh (x \pm \sqrt{d} t) . \\
& v(x, t)= \pm \sqrt{d} \tanh ^{2}(x \pm \sqrt{d} t) .
\end{aligned}
$$

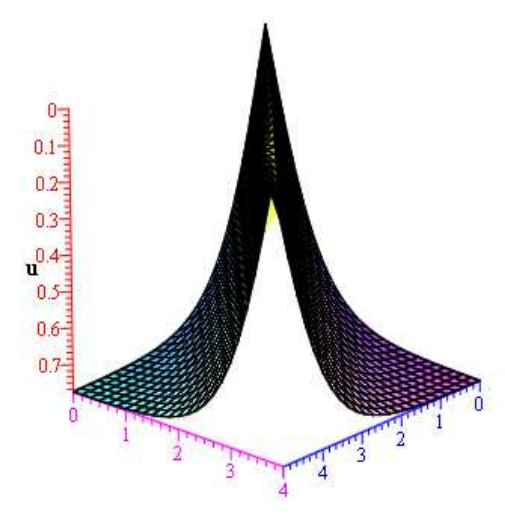

Fig. 1: Bell shaped profile of Eq. (16) with $d=$ 0.60 .

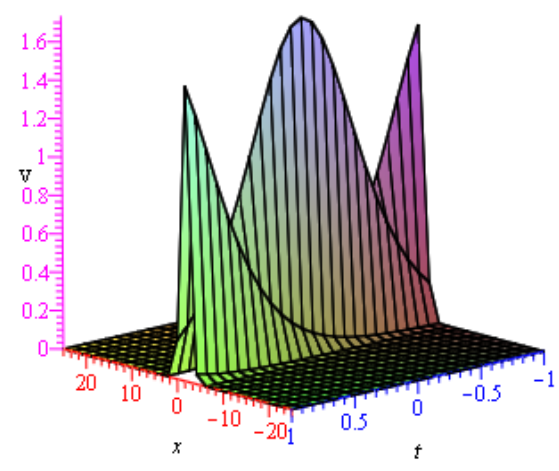

Fig. 3: Profile of Eq. (16) with with $d=3$, $-25 \leq x \leq 25,-1 \leq t \leq 1$.

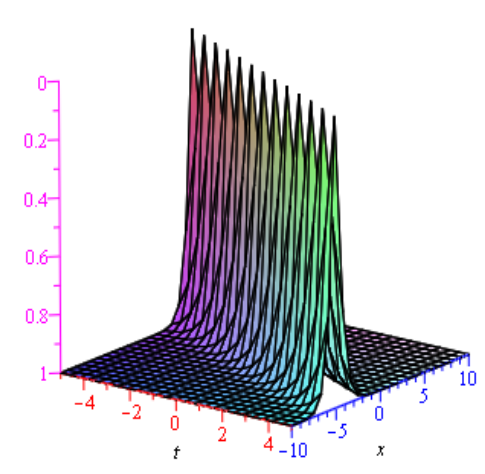

Fig. 2: Profile of Eq.(17) with $d=1$.

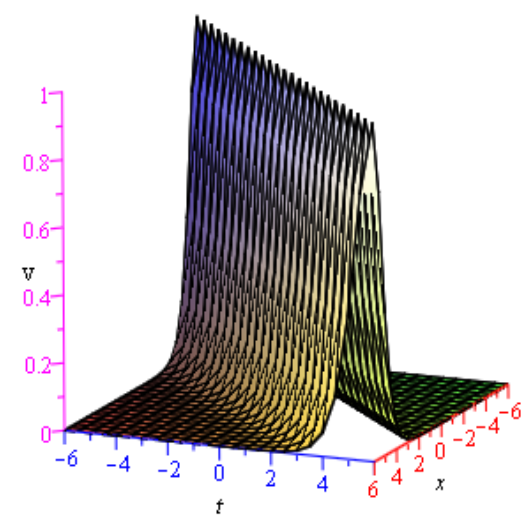

Fig. 4: Bell shaped profile of (17) with $d=1$, $-6 \leq x \leq 6,-6 \leq t \leq 6$. 


\subsection{The extended tanh-function method}

In this subsection we will apply the extended tanh-function method to find the exact traveling wave solutions of KonnoOono equation for the value of $m=1$, the finite expansion of extended tanh-function method admits,

$$
u(x, t)=a_{0}+a_{1} Y+\frac{b_{1}}{Y} .
$$

Now, Substituting Eq. (18) into Eq. (14), and equating the coefficients of the powers $Y$ then we obtain a system of algebraic equations:

$$
\begin{aligned}
& 2 a_{1}^{3}+52 \omega^{2} a_{1}=0 \\
& 6 a_{0} a_{1}^{2}=0 \\
& 6 a_{1}^{2} b_{1}+2 d a_{1}-2 \omega^{2} a_{1}+6 a_{0}^{2} a_{1}=0 \\
& 2 d a_{0}+2 a_{0}^{3}+12 a_{0} a_{1} b_{1}=0 \\
& 6 a_{0}^{2} b_{1}-2 \omega^{2} b_{1}+2 d b_{1}+6 a_{1}^{2} b_{1}^{2}=0 \\
& 6 a_{0} b_{1}^{2}=0 \\
& 2 \omega^{2} b_{1}+2 b_{1}^{3}=0 .
\end{aligned}
$$

Solving these algebraic equations for $a_{0}, a_{1}, a_{2}, b_{1}$ and $\omega$ then we obtain following sets of solutions:

Set-1 $\omega= \pm \sqrt{d}, a_{0}=a_{1}=0, b_{1}= \pm I \sqrt{d}$

Set-2 $\omega= \pm \sqrt{d}, a_{0}=b_{1}=0, a_{1}= \pm I \sqrt{d}$

Set-3 $\omega= \pm \frac{1}{2} \sqrt{d}, a_{0}=0, a_{1}= \pm \frac{1}{2} I \sqrt{d}, b_{1}= \pm \frac{1}{2} I \sqrt{d}$

Set-4 $\omega= \pm \frac{1}{\sqrt{ } 2} I \sqrt{d}, a_{0}=0, a_{1}= \pm \frac{1}{\sqrt{2}} \sqrt{d}, b_{1}=\mp \frac{1}{\sqrt{2}} \sqrt{d}$.

By solving these sets of equations we required the following solutions:

$$
\begin{gathered}
u_{1}= \pm I \sqrt{d} \operatorname{coth}(x \mp \sqrt{ } d t) \\
v_{1}=\mp \sqrt{ } d\left(\operatorname{coth}^{2}(x \mp \sqrt{ } d t)-1\right) \\
u_{2}= \pm I \sqrt{d} \tanh (x \mp \sqrt{ } d t) \\
v_{2}=\mp \sqrt{ } d(\tanh 2(x \mp \sqrt{ } d t)-1) \\
u_{3}= \pm \frac{1}{2} I \sqrt{ } d\left(\tanh \left(x \mp \frac{1}{2} \sqrt{ } d t\right) \pm \operatorname{coth}\left(x \mp \frac{1}{2} \sqrt{ } d t\right)\right) \\
v_{3}=\mp \frac{1}{2} \sqrt{ } d\left(\left(\tanh \left(x \mp \frac{1}{2} \sqrt{ } d t\right) \pm \operatorname{coth}\left(x \mp \frac{1}{2} \sqrt{ } d t\right)\right)^{2}-4\right) \\
u_{4}= \pm \frac{1}{\sqrt{ } 2} \sqrt{ } d\left(\tanh \left(x \mp \frac{1}{\sqrt{2}} I \sqrt{ } d t\right) \mp \operatorname{coth}\left(x \mp \frac{1}{\sqrt{2}} I \sqrt{ } d t\right)\right) \\
\frac{1}{\sqrt{2}} I \sqrt{ } d\left(\left(\tanh \left(x \mp \frac{1}{\sqrt{2}} I \sqrt{ } d t\right) \mp \operatorname{coth}\left(x \mp \frac{1}{\sqrt{2}} I \sqrt{ } d t\right)\right)^{2}+2\right) .
\end{gathered}
$$




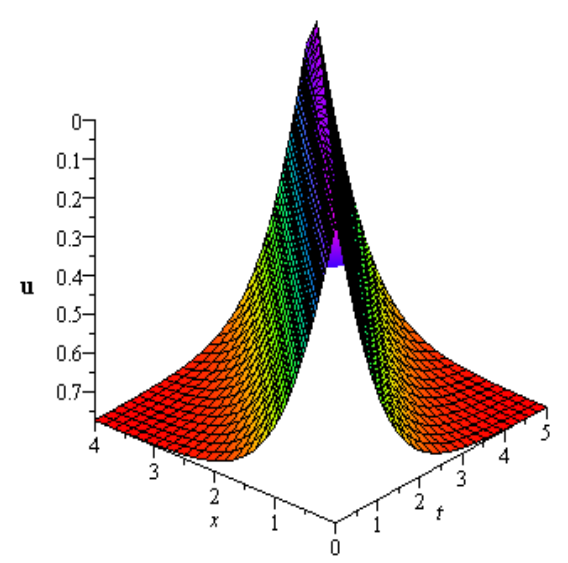

Fig. 5: Bell shaped wave of Eq. (21) with $d=$ $0.60,0 \leq x \leq 4,0 \leq t \leq 5$.

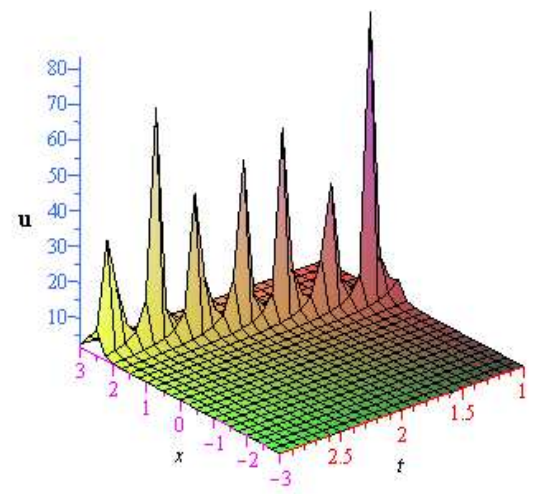

Fig. 7: Modulus plot of Eq. (23) with $d=3$, $-3 \leq x \leq 3,1 \leq t \leq 3$.

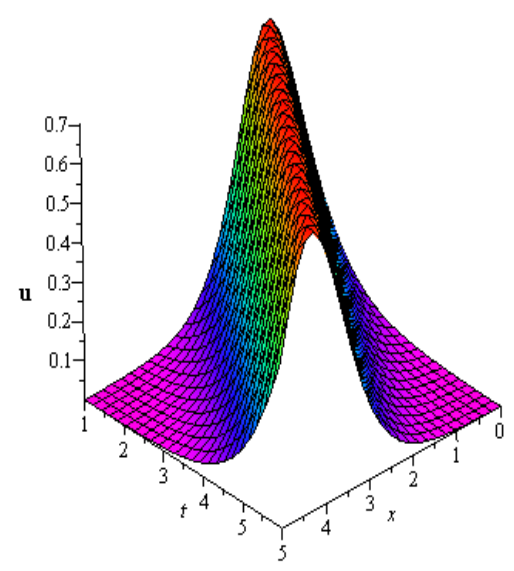

Fig. 6: Bell shaped soliton, graph of Eq.(22) with $d=0.50,0 \leq x \leq 5,1 \leq t \leq 6$.

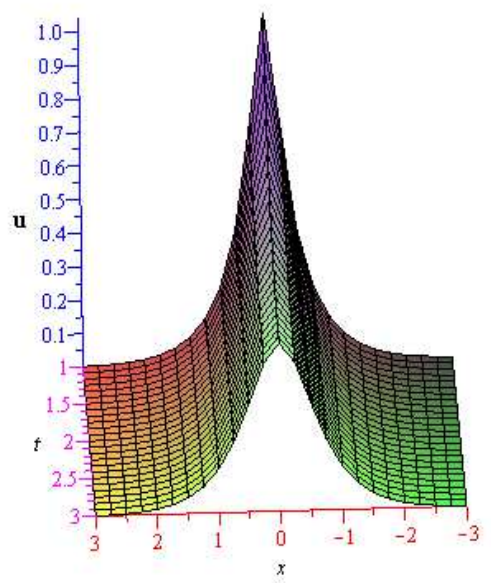

Fig. 8: Modulus plot of Eq. (25) with $d=1 / 8$, $-3 \leq x \leq 3,1 \leq t \leq 3$.

\section{Conclusions}

The tanh-function method and extended tanh-function method have been applied successfully to construct the exact traveling wave solutions of new coupled Konno-Oono Equation. The traveling wave transformation formulae have used to find the solutions. We have emphasized in this work that this relevant transformation is powerful and can be effectively used to discuss nonlinear evolution equations and related models in scientific fields. 


\section{References}

[1] J.H. He, X.H. Wu, Exp-function method for nonlinear wave equations, Chaos, Solitons ～\& Fractals 30: 700-708 (2006).

[2] M.A. Akbar, NHM. Ali, Exp-function method for Duffing Equation and new solutions of $(2+1)$ dimensional dispersive long wave equations, Progress in Applied Mathematics, 1 (2), 30-42 (2011).

[3] H. Naher, A.F. Abdullah, M.A. Akbar,The Exp-function method for new exact solutions of the nonlinear partial differential equations, International Journal of Physical Sciences, 6 (29), 6706-6716 (2011).

[4] H. Naher, AF. Abdullah, MA. Akbar, New traveling wave solutions of the higher Dimensional nonlinear partial differential equation by the Exp-function method, Journal of Applied Mathematics, volume 2012, (doi: 10.1155(2012)575387) (2012).

[5] A. Bekir, A. Boz, Exact solutions for nonlinear evolution equations using Exp-function method, Physics Letter A, 372, 1619-1625 (2008).

[6] R. Hirota, Exact envelope soliton solutions of a nonlinear wave equation, Journal of Mathematical Physics,14, 805-10 (1973).

[7] R. Hirota, J. Satsuma, Soliton solutions of a coupled KDV equation, Physics Letter A,85, 404-408 (1981).

[8] G. Adomian, Solving frontier problems of physics: The decomposition method, Boston, M A: Kluwer Academic, (1994).

[9] M. S. Islam, K. Khan, M. A. Akbar, and A. Mastroberardino, A note on improved F-expansion method combined with Riccati equation applied to nonlinear evolution equations, Royal Society Open Science, 1, 140038 (doi.org/10.1098/rsos.140038) (2014).

[10] Sirendaoreji, New exact travelling wave solutions for the Kawahara and modified Kawahara equations, Chaos Solitons Fractals, 19, 147-150 (2004).

[11] M. Shakeel, and S. T. Mohyud-Din,A Novel $\left(G^{\prime} / G\right)$-Expansion Method and its Application to the (3+ 1)-Dimensional Burger's Equations, International Journal of Applied \& Computational Mathematics, 2(1), 13-24 (DOI 10.1007/s40819-015-0039-5), (2016).

[12] K. Khan, M. A. Akbar, and H. Koppelaar, Study of Coupled Nonlinear Partial Differential Equations for finding Exact Analytical Solutions. Royal Society Open Science, 2, 140406, (http://dx.doi.org/10.1098/rsos.140406), (2015).

[13] K. Khan, and M. A. Akbar, Study of analytical method to seek for exact solutions of variant Boussinesq equations. SpringerPlus, 3,324, (DOI:10.1186/2193-1801-3-324), (2014).

[14] M. H. Islam, K. Khan, M. A. Akbar, and M. A. Salam, Exact Traveling Wave Solutions of Modified KdV-Zakharov-Kuznetsov Equation and Viscous Burgers Equation, SpringerPlus, 3,105, (doi:10.1186/2193-1801-3-105), (2014).

[15] M. E. Islam, K. Khan, M. A. Akbar and R. Islam, Traveling Wave Solutions Of Nonlinear Evolution Equation Via Enhanced $\left(G^{\prime} / G\right)$-Expansion Method, GANIT: Journal of Bangladesh Mathematical Society, 33, 83-92, (http://dx.doi.org/10.3329/ganit.v33i0. 17662), (2013).

[16] K. Khan, and M. A. Akbar, Solitary Wave Solutions of Some Coupled Nonlinear Evolution Equations. Journal of Scientific Research, 6(2), 273-284. (doi.org/10.3329/jsr.v6i2.16671), (2014).

[17] M. T. Ahmed, K. Khan and M. A. Akbar, Study of Nonlinear Evolution Equations to Construct Traveling Wave Solutions via Modified Simple Equation Method. Physical Review \& Research International, 3(4), 490-503 (2013).

[18] W. Malfliet and W. Hereman, The tanh method: A tool for solving certain classes of Nonlinear Evolution and Wave Equations, Physica Scripta, 54, 563-568 (1996).

[19] X. D. Zheng, T. C Xia and H. Q. Zhang, New exact travelling wave solutions for compound KdV-burgers equation in mathematical physics, Appl. Math. E-Note, 2, 45-50 (2002).

[20] J. Manafian, M. Lakestani, and A. Bekir, Study of the Analytical Treatment of the (2+1)-Dimensional Zoomeron, the Duffing and the SRLW Equations via a New Analytical Approach. International Journal of Applied \& Computational Mathematics, pp1-26, (DOI 10.1007/s40819-015-0058-2), (2015).

[21] A M.Wazwaz, The extended tanh method for new soliton solutions for many forms of the fifth-order KdV equations, Applied Mathematics and Computation, 184 (2), 1002-1014, (doi:10.1016/j.amc.2006.07.002), (2007).

[22] A M.Wazwaz, New solitary wave solutions to modified forms of Degasperis-Procesi and Camassa-Holm equations, Applied Mathematics and Computation, 186(1),130-141, (doi:10.1016/j.amc.2006.07.092), (2007).

[23] M Labidi, H Triki, E V Krishnan and A Biswas, Soliton solutions of the long-short wave equation with power law nonlinearity, Journal of Applied Nonlinear Dynamics 1 (2), 125-140 (2012). 
[24] K. Konno and H. Oono, New coupled integrable dispersionless equations, Journal of the Physical Society of Japan, vol. 63, pp. 377-378, (1994). 